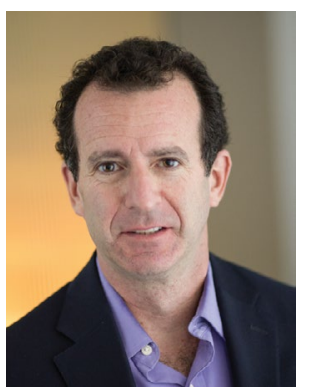

\title{
Will this pandemic be the catalyst to finally reform humanitarian responses?
}

\author{
The COVID-19 pandemic should revive a shared understanding of humanitarian emergencies \\ and crisis resolution, opening the door to transformative change in humanitarian responses. \\ But it has also revealed political opportunism and poor data-reporting structures.
}

F or the first time since the influenza pandemic in 1918, the whole world has been directly affected by the COVID-19 pandemic, a global humanitarian emergency. Most of us had little input into how decisions were made that dramatically affected our lives and livelihoods. This helplessness and lack of agency are often how people affected by humanitarian emergencies feel every day. As with any crisis, there are opportunities for learning and making positive changes.

An obvious lesson for those working in humanitarian emergencies is that a lack of quality data has blinded us to the consequences of the pandemic in humanitarian settings. Many seasoned humanitarians believed that humanitarian populations would disproportionally be affected by this pandemic due to poor baseline health, a high density of people living in squalid conditions with limited ability to physically distance, and fledgling water and sanitation services. But the reported transmission of COVID-19 and hospitalizations and deaths due to COVID19 among people affected by humanitarian emergencies do not reflect those predictions and are lower than expected.

However, it is too early to interpret this information as good news. It is possible that a younger demographic composition of populations, mostly outdoor interactions due to favorable weather conditions, fewer comorbidities, and other factors could explain this discrepancy. It is also possible that wide-scale transmission and death have gone undocumented. Inadequate testing, poorly implemented contact tracing, inadequate mortality reporting, and limited seroprevalence surveys have frustrated our understanding of the current situation and have hampered our ability to predict and respond.

As with other large-scale epidemics, there are growing concerns that an increase in indirect morbidity and mortality due to the focus on response to COVID-19 and mitigation measures may have immediate and long-lasting consequences. During the Ebola epidemics in West Africa, the number of maternal, neonatal and malarial deaths increased. Here again, a paucity of data thwarts efforts to document and respond to the indirect effects of the COVID-19 pandemic in humanitarian settings.

Moreover, the world witnessed deliberate and calculated responses by governments to abuse COVID-19 public-health orders to further anti-refugee and anti-migrant agendas. In March 2020, the US government ordered the closure of its land borders to migrants and asylum seekers while keeping them open for Americans, green-card holders and commercial traffic, together with numerous other exceptions; such restrictions violated US and international refugee law. European Union member states, such as Greece and Hungary, also used public-health orders to reduce or stop asylum, due to the COVID-19 pandemic, while still allowing other groups to enter their countries. Such policies, implemented under the guise of public health, are not evidence based and are against international refugee law. This should not be tolerated.

An interesting consequence of this pandemic is that it may finally provide space for humanitarian responses to become more localized. One aspect of 'localization' would mean that United Nations agencies and international non-governmental organization (NGOs) would receive much less funding, which would ultimately affect their reach, size, power and influence. Localization would also further the 'decolonialization' of humanitarian assistance, an important concept that is now being openly debated and connected with other movements such as Black Lives Matter. The humanitarian community was unable to flood countries with expatriate humanitarian workers; they helped mainly from their homes. Perhaps the pandemic can accelerate some of the goals of the Grand Bargain, agreed upon at the May 2016 World Humanitarian Summit, such as providing at least $25 \%$ of humanitarian funding directly to national responders by 2020; this and other goals have not been met.

Looking forward, the humanitarian community could use the COVID-19 pandemic as a catalyst to undertake much-needed reforms.

There must be a commitment to share data, in a confidential and secure manner, among United Nations agencies, NGOs and academic institutions to allow increased understanding of diverse humanitarian contexts. Poor data quality and lack of sharing have impeded our understanding of the effects of the pandemic in most humanitarian contexts and have consequently limited our planning and response. More funds should be provided directly to affected governments and national NGOs, and the voices of people affected by humanitarian emergencies should direct the response to the crisis. There should be greater recognition by the international community and a collective responsibility for the inclusion of non-nationals, including migrants, asylum seekers, refugees, stateless people, in the current pandemic. Vaccine nationalism means that non-nationals will probably be excluded from receiving vaccines against COVID-19, at least for now. Commitments, as outlined in refugee and migration global compacts, should become legally binding, and abuse via public-health orders that target migrants and refugees must explicitly be banned.

This pandemic could finally be the impetus for the humanitarian community to truly listen to and work with people affected by humanitarian emergencies, and to provide them with the dignity, respect and autonomy that will allow them to make their own decisions about their future. It could also be the catalyst to ensure that governments and national NGOs directly receive donor funds to provide a culturally appropriate response in a cost-effective manner. Such reforms need to occur now, as future conflict, displacement and pandemics will occur more frequently as the effects of the climate crisis accelerate.

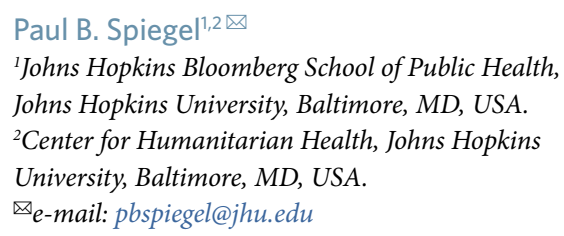

